\title{
Lack of long-lived quantum coherence in the photosynthetic energy transfer
}

\author{
Hong-Guang Duan ${ }^{1,2,3}$, Valentyn I. Prokhorenko ${ }^{1}$, Richard J. Cogdell ${ }^{4}$, Khuram Ashraf $^{4}$, \\ Amy L. Stevens ${ }^{1,3,5}$, Emilie Wientjes ${ }^{6}$, Roberta Croce $^{6}$, Michael Thorwart ${ }^{2,3}$ and $R$. J. \\ Dwayne Miller ${ }^{1,3,5}$ \\ ${ }^{1}$ Max Planck-Institute for the Structure and Dynamics of Matter, Luruper Chaussee 149, 22761 \\ Hamburg, Germany \\ ${ }^{2}$ I. Institut für Theoretische Physik, Universität Hamburg, Jungiusstraße 9, 20355 Hamburg, Germany \\ ${ }^{3}$ The Hamburg Center for Ultrafast Imaging, Luruper Chaussee 149, 22761 Hamburg, Germany \\ ${ }^{4}$ Institute of Molecular, Cell, and Systems Biology, College of Medical, Veterinary, and Life Science, \\ University of Glasgow, Glasgow G128QQ, United Kingdom \\ ${ }^{5}$ The Departments of Chemistry and Physics, University of Toronto, 80 St. George Street, Toronto, \\ M5S 3H6 Canada \\ ${ }^{6}$ Department of Physics and Astronomy and Institute for Lasers, life and Biophotonics, Faculty of \\ Sciences, VU University Amsterdam, DeBoelelaan, 1081, HV Amsterdam, The Netherlands
}

\begin{abstract}
We have studied the FMO, LHCII and PSII reaction center complex by electronic 2D spectroscopy. At ambient temperature the electronic coherences are too short lived to play any functional role in the natural energy transfer.
\end{abstract}

\section{Introduction}

In the initial steps of natural light-harvesting, pigment-protein complexes (PPCs) complete the light-energy transfer and charge separation with near unity quantum efficiency. To investigate the energy transfer dynamics, one of the most typical PPCs, the FennaMatthews-Olson (FMO), has been selected. It is found in low light-adapted green sulfur bacteria. Its task is the energy transport between the peripheral chlorosome and the reaction center. It was the first PPC to be structurally analyzed by X-ray crystallography [1]. The complex is a trimer made of identical subunits, each of which contains eight bacteriochlorophyll a (Bchla) molecules (see the left side of Fig. 1). By virtue of its relatively small size, it provides a very important model for the study of natural excitation energy transfer (EET) and has been extensively studied experimentally and theoretically. In 1997, Savikhin et al. [2] have observed quantum beating in the FMO complex at low temperature using one-color pump-probe spectroscopy. Recently, the $2 \mathrm{D}$ electronic spectra of the FMO complex were measured at 77K [3] and room temperature [4] and long-lived oscillations were reported. Their origin was interpreted to be electronic quantum coherence generated by the excitonic couplings between the pigments. Moreover, coherence was reported for the other PPCs as well [5]. It was found that some oscillations are robust 
against increasing temperature. However, the origin of these long-lived oscillatory signals is still under debate. In fact, as an alternative explanation, the so called vibrationally-

assisted quantum coherence has been proposed [6,7]. In order to clarify the nature of these oscillations, we have performed 2D spectroscopic measurements of the FMO complex at ambient temperature and the associated theory was developed. No long-lived quantum coherence and associated quantum beatings were identified, in agreement with previous path-integral simulations [8]. Moreover, we have also performed the measurements of LHCII and the PSII reaction center, which are the typical light-harvesting complexes of higher plants. All the measurements show no evidence of long-lived electronic coherence.

\section{Experimental setup}

Ultrashort light pulses were generated by a home-built non-collinear optical parametric amplifier. It was tuned to the near infrared and the spectral center is located at $800 \mathrm{~nm}(650$ $\mathrm{nm}$ for LHCII and reaction center) with a FWHM of $100 \mathrm{~nm}$. The generated pulse was compressed to $16 \mathrm{fs}$ by a combination of a prism pair (F2) and a deformable mirror $(19 \mathrm{x} 4$ actuators, OKO Technologies). No chirp was detected using transient-grating FROG. The spectra were measured by the 2D spectrometer with the configuration described in Ref. [9]. The spectra were collected at each fixed waiting time $\mathrm{T}$ by scanning the delay time. Dependent on the sample, 120-250 spectra have been averaged at each delay point. The waiting time $\mathrm{T}$ was linearly scanned within $0-2 \mathrm{ps}$ in steps of 10 (25) fs and with logarithmically spaced steps of up to $10 \mathrm{ps}$ ( $80 \mathrm{ps}$ for PSII reaction center). The FMO trimer was isolated from green sulfur bacteria $(\mathrm{Cl}$. tepidum). LHCII trimers and PSII reaction center were isolated from spinach. Before the measurement, the sample was filtered with a 0.2 micrometer filter in order to reduce light scattering.

\section{Results and discussion}

In the right panel of Fig. 1, we show the agreement of the measured and the calculated 2D electronic spectra at different waiting times. At zero waiting time, the $2 \mathrm{D}$ spectrum is significantly elongated along the diagonal direction, which manifests strong inhomogeneous broadening. Moreover, the anti-diagonal bandwidth of the center peaks has been estimated to $175 \mathrm{~cm}^{-1}$, which corresponds to the lifetime of the electronic dephasing of $\sim 60$ fs. With increasing waiting time, the peak amplitude is significantly decreased due to the dissipation from the environment.
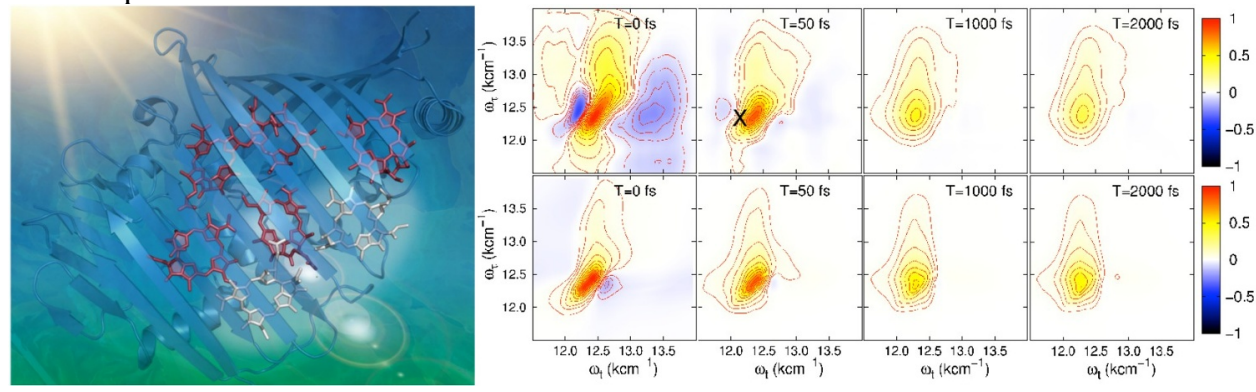

Fig. 1. Left: Molecular structure of the FMO protein complex. Right top: Representative 2D spectra measured at room temperature at different waiting times $\mathrm{T}$ as indicated. Right bottom: Calculated 2D electronic spectra at the same waiting times. 
In order to resolve the lifetime of electronic coherence, the time-evolved magnitude of a selected peak (marked as "X" in Fig. 1) is shown in the left panel of Fig. 2. No long-lived oscillations are observed, which agrees with a short lifetime for electronic dephasing. For a complete study, we have measured the 2D spectra of LHCII and the PSII reaction center. All show a similar timescale of the electronic coherence, which is summarized in the table at the right panel of Fig. 2. Based on these systematic studies, we confirm that the short lifetime of electronic coherence prevents the development of long-lived oscillations related to exciton coherence, and is unimportant with respect to the energy transfer in the typical photosynthetic protein complexes at ambient temperature. The observed decoherence dynamics indicate that energy transport involves partially delocalized excitons executing an incoherent transport process.

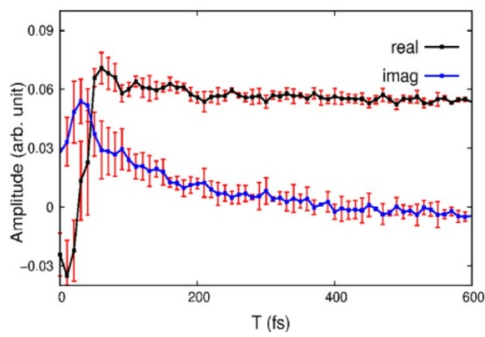

\begin{tabular}{|l|c|c|}
\hline sample & Lifetime (fs) & Reference \\
\hline LHCII & $\sim 65 \mathrm{fs}$ & JPCB 119, 12017 (2015). \\
\hline $\begin{array}{l}\text { Reaction } \\
\text { center }\end{array}$ & $\sim 56 \mathrm{fs}$ & Sci. Rep. 7, 12347 (2017). \\
\hline $\begin{array}{l}\text { Indocarbocyanine } \\
\text { dimer }\end{array}$ & $\sim 50 \mathrm{fs}$ & $\begin{array}{l}\text { New J. Phys. 17, 072002 (2015). } \\
\text { Nat. Chem. 6, 196 (2014). }\end{array}$ \\
\hline FMO & $\sim 60 \mathrm{fs}$ & PNAS 114, 8493 (2017). \\
\hline
\end{tabular}

Fig. 2. Left: Time-evolved amplitude (real and imaginary) of the selected peak " $X$ " in Fig. 1. Right: Lifetime of the electronic coherence in the LHCII, PSII reaction center, indocabocyanine dimer and FMO protein complexes.

\section{References}

1. R.E. Fenna, B.W. Matthews, Nature 258, 573 (1975).

2. S. Savikihin, D.R. Buck, W.S. Struve, Chem. Phys. 223, 303 (1997).

3. G.S. Engel, T.R. Calhoun, E.L. Read, T.K. Ahn, T. Mancal, Y.C. Cheng, R.E. Blankenship, G.R. Fleming, Nature 446, 782 (2007).

4. G. Panitchayangkon, D. Hayes, K.A. Fransted, J.R. Caram, E. Harel, J.A. Wen, R.E. Blankenbship, G.S. Engel, Proc. Natl. Acad. Sci. 107, 12766 (2010).

5. G.D. Scholes, G.R. Fleming, A. Olaya-Castro, R. van Grondelle, Nat. Chem. 3, 763 (2011).

6. A.W. Chin, J. Prior, R. Rosenbach, F. Caycedo-Soler, S. F. Huelga, M. B. Plenio, Nat. Phys. 9, 113 (2013).

7. A. Halpin, P.J.M. Johnson, R. Tempelaar. R.S. Murphy, J. Knoester, T.L.C. Jansen, R.J.D. Miller, Nat. Chem. 6, 196 (2014).

8. P. Nalbach, D. Braun, M. Thorwart, Phys. Rev. E 84, 041926 (2011).

9. V.I. Prokhorenko, A. Halpin, R.J.D. Miller, Opt. Exp. 17, 9764 (2009). 